\title{
Pathologic diagnosis in head and neck practice: how fast is fast enough?
}

\author{
Kenneth O. Devaney • Alessandra Rinaldo • \\ Alfio Ferlito
}

Received: 14 September 2009 / Accepted: 16 September 2009 / Published online: 26 September 2009

(C) Springer-Verlag 2009

\section{Introduction}

Diagnosis of pathologic specimens has become faster over the past decade or so; nevertheless, differences in the speed of diagnosis remain. This article asks a simple question: just what is a reasonable expectation for the turnaround time for a routine pathologic diagnosis? Should it be measured in weeks, days, hours or minutes?

There was a time, not awfully long ago, when the rendering of a pathologic diagnosis could be a relatively leisurely affair: the time from the receipt of a tissue specimen to a final written pathology report could span the range from a few days to a few weeks (in the case of particularly puzzling specimens). Those days are gone forever.

Today, there are large commercial laboratories promising same-day service for many types of pathology specimens, while teaching hospital laboratories are somewhat slower. Is one approach better than the other?

\section{The gold standard-frozen section}

Mayo Clinic in Rochester, Minnesota, has raised the process of rendering lightning-fast surgical pathology diagnoses to a real art form [1-3]. Surgeons removing tumors in

K. O. Devaney

Department of Pathology,

Allegiance Health, Jackson, MI, USA

A. Rinaldo · A. Ferlito $(\square)$

Department of Surgical Sciences, ENT Clinic,

University of Udine, Piazzale S.Maria della Misericordia,

33100 Udine, Italy

e-mail: a.ferlito@uniud.it their operating rooms can expect a relatively comprehensive pathologic diagnosis within 10 or $15 \mathrm{~min}$ in most instances, courtesy of frozen section examination. While permanent sections are examined the following day for confirmation of the original frozen section diagnosis, as a practical matter a thorough pathologic examination is reported and available in the patient's medical record before the patient leaves the operating room to go to the recovery room.

As attractive as this process is, it is unlikely to be reproduced widely. One substantial hurdle is posed by the very nature of frozen section examination; and that is its artifactual overlay. As in microscopic images, sections prepared by frozen section are by their very nature less clear than are permanent sections, that is, a trade-off is accepted between image quality and speed of diagnosis. In return for some diminution of image clarity, Mayo Clinic pathologists return diagnoses within a matter of a very few minutes.

As appealing as this is, there are some very real hurdles to extending this practice everywhere. There is a technical dimension: Mayo uses a particular specialized type of freezing microtome, a different piece of equipment from the conventional cryostats employed in most hospital laboratories today. The widely used cryostats freeze tissue a little more slowly than the Mayo's freezing microtome and are subject to some artifacts that are not as frequently encountered with the freezing microtome (this latter instrument, for example, is better able to handle adipose tissue than is the widely available cryostat). However, the freezing microtome, with its enclosed refrigerant, is a highly specialized piece of equipment, both expensive to construct and expensive to operate and particularly labor intensive in its daily usage.

There is a professional limitation as well: Mayo Clinic pathologists train over the years to interpret their frozen 
section specimens. By contrast, pathologists elsewhere are grounded in the daily interpretation of permanently prepared images for most of their diagnostic work. This means that frozen section interpretation often becomes something in which they do not spend the bulk of their time practicing, and so are not as experienced as are Mayo Clinic pathologists when it comes to interpreting frozen section specimens.

There are, finally, some potential limitations to the freezing of the tissue itself. Ancillary techniques for diagnosis and prognosis, such as immunohistochemistry or molecular studies, have in the main been refined for application to routinely fixed tissues. It is true that frozen tissue methods have been developed for use with many of the antibodies used in immunohistochemistry, and so this is not an insurmountable problem. Nevertheless, an abrupt shift to frozen section processing of tissues would necessitate a re-evaluation of procedures for the ancillary usage of tissues for a wide range of other uses, which would likely not be a trivial matter.

When Mayo Clinic expanded beyond Minnesota to Arizona and Florida in the 1980s, it became apparent that it was actually very difficult to reproduce the frozen section procedures at work in Rochester in those two sites: the expense of recreating the original procedures and practices proved to be prohibitive. This, in turn, suggests that replacing current conventional pathologic practices with Mayo Clinic Rochester-style frozen section practices would not be a practical means of speeding the pathologic diagnostic process along.

\section{The present options}

The processing of human tissue for pathologic diagnosis ordinarily begins with fixation, a process that halts cell death and dissolution. This process of fixation requires some finite amount of time, usually measured in hours; larger portions of tissue require longer periods of fixation, while tiny portions of tissue are fixed much more rapidly.

After fixation, this same tissue must be made hard enough to cut into exceedingly thin sections that will transmit light. This process of embedding, which usually rests upon infiltration of the tissue with melted paraffin, also requires a period of several hours. Finally, after cutting these thin sections, the sections must be transferred to individual glass slides, stained and coverslipped.

Taken together, the time from the initial dissection of the specimen to the production of routinely stained glass slides may range from 9 to $15 \mathrm{~h}$.

What can be done to accelerate this process? For one thing, the production process could be started earlier in the day, so as to produce stained slides at midnight, or at 3 a.m.
As well, surgical pathology laboratories could be staffed on weekends and holidays. This may not be such a boon, of course, there are few clinicians to be found in their offices at midnight or 3 a.m., or on a Sunday or a holiday, and so this sort of solution does not seem likely to win too many supporters.

Another approach would be to experiment with an alternative to the time-honored tradition of batch processing specimens, in which the entire day's work is processed by an automated process at the end of the work day, after the workers have left for the day. An alternative, then, might be to construct a continuous system for processing specimens as they are received, without the need for waiting for a full day's "batch" to accumulate.

As it happens, manufacturers are beginning to market instruments designed to do just that: produce slides via a continuous throughput process; this, in turn, is coupled with microwave-assisted tissue processing, which serves to accelerate the overall process yet further [4-7].

This not so modest change, from daily batching to continuous processing, will not be an effortless change to put into effect. The equipment will be expensive, and the personnel who work with it may be asked to carry out different tasks than they presently do (in preparing sections for rapid processing, for example, the sections must be thinner than are the sections used in conventional processing). Still, these instruments do seem to produce specimens faster than routine processing does, and so they may be worth the expense for those laboratories that can exploit their capabilities. As noted above, a laboratory able to provide diagnoses at midnight, and a clinician population ready to receive those midnight diagnoses, may be quite pleased with a microwave-assisted continuous throughput instrument. Otherwise, having diagnostic pathology work ready to go at a time when neither pathologists nor surgeons are available to handle it would seem pointless.

\section{The future}

There will also always be a difference between an academic center and a for-profit company offering diagnostic pathology services. While the latter is free to focus on the crafting of reports as an endpoint, the former, the academic center, has twin purposes: to produce reports and also to train the next generation of diagnostic surgical pathologists. To this end, there is some finite time required for the trainee pathologists to perform their own examinations and reach their own conclusions, before reviewing their findings with their attendings. Some academic centers have begun telescoping these functions, obliging trainees and attendings to review their cases simultaneously; but there seems to be something lost in such a scheme: the trainees run the risk of 
parroting what they are told, without having to form and then defend their own opinions. Still, this may be the way of the future, and so may not be subject to debate; time will tell.

In any event, it seems as though a few expectations can be sketched out for at least the foreseeable future. Knowing that there is a finite time required to process tissues for diagnosis, the partnership between those obtaining tissues for pathologic examination, and those actually carrying out those pathologic examinations, may grow closer. For example, knowing that tissues obtained late on a Friday or Saturday evening will not be reported out until the subsequent Monday, surgeons may prefer to schedule their cases for earlier in the week. If speed of reporting is of paramount importance, as for example, with a needle biopsy from a suspected breast malignancy of a patient who is extremely anxious, the surgeon may perform that biopsy earlier in the morning (rather than at the end of the workday), to assure that it is indeed processed with that day's work and reported out the next day. Alternatively, of course, the surgeons may lobby for $24 \mathrm{~h} / 7$ day a week staffing of pathology laboratories. While labor shortages may limit the practicality of the latter idea, it is true that some private commercial laboratories selling diagnostic services built around a narrow niche market (just gastrointestinal biopsies, for example, or just urologic specimens) are beginning to offer expanded hours, with diagnoses being made increasingly "after hours." In part, this strategy seems designed to combat the increase in turnaround time that would otherwise be forced by the time invested in collecting specimens from across a broad service area and then transporting them to a central site for final processing and diagnosis.

As a general rule, biopsies received by the laboratory (and this means received at the working bench, not merely dropped off at some remote collection site) by a set hour (say, 3, 4 or 5 p.m., depending on that laboratory's staffing) should ordinarily be expected to result in the production of a pathology report the next working day. Should additional fixation and dissection be required (as for a particularly large and complex specimen), then an additional day may be added. Should the tissue be mineralized and so require decalcification, this too may add a day or two to the point at which a report could reasonably be expected. Should additional studies (e.g., immunohistochemistry or molecular studies) be required, then an additional day for immunohistochemical studies (several days for certain molecular investigations) should be anticipated. Increasingly, laboratories are producing preliminary reports based on routine light microscopy, with the promise of additional information in the next several days.

At the risk of oversimplifying, a small biopsy specimen, e.g., a needle biopsy specimen, received before some set hour on a Monday afternoon should result in the production of a pathology report on Tuesday. A large, anatomically complex surgical resection specimen dropped off in the pathology laboratory late on Monday may require additional fixation overnight, with production of a pathology report by Wednesday or Thursday. These rough guidelines would clearly be subject to local variations, where laboratory support personnel cannot process a day's work on the same day, and the process of batching work may occur less than daily, slowing things down. Where consultation among pathologists is judged to be necessary before the rendering of a final diagnosis, there again may be several days (or depending on the availability of consultants, even weeks) spent in awaiting a final pathology report.

These rough guidelines should allow clinicians to make plans that incorporate "pathology time" into the time they already allot to work with their patients. In an individual practice, there really is no substitute for the clinician having a relationship with his or her pathologist(s). It is those pathologists who are in the very best position to help that clinician strategize about what would happen next in the patient's care: not just the "what," but the "when" as well.

\section{References}

1. Wilson LB (1905) A method for the rapid preparation of fresh tissues for the microscope. JAMA 45:1737

2. Dahlin DC (1980) Seventy-five years' experience with frozen sections at the Mayo Clinic. Mayo Clin Proc 55:721-723

3. Keeney G, Leslie K (2008) Preparing fresh tissues for the microscope. JAMA 300:1074-1076

4. Morales AR, Nassiri M, Kanhoush R, Vincek V, Nadji M (2004) Experience with an automated microwave-assisted rapid tissue processing method: validation of histologic quality and impact on the timeliness of diagnostic surgical pathology. Am J Clin Pathol 121:528-536

5. Vernon S (2005) Continuous throughput rapid tissue processing revolutionizes histopathology workflow. Lab Med 36:300-302

6. Panja P, Sriram G, Saraswathi TR, Sivapathasundharam B (2007) Comparison of three different methods of tissue processing. J Oral Maxillofac Pathol 11:15-17

7. Munkholm J, Talman ML, Hasselager T (2008) Implementation of a new rapid tissue processing method-advantages and challenges. Pathol Res Pract 204:899-904 